TITLE:

\title{
Initial Kaluza-Klein fluctuations and inflationary gravitational waves in braneworld cosmology
}

AUTHOR(S):

Kobayashi, T

CITATION:

Kobayashi, T. Initial Kaluza-Klein fluctuations and inflationary

gravitational waves in braneworld cosmology. PHYSICAL REVIEW D 2006, 73(12): 124031.

\section{ISSUE DATE:}

2006-06

URL:

http://hdl.handle.net/2433/49924

RIGHT:

Copyright 2006 American Physical Society 


\title{
Initial Kaluza-Klein fluctuations and inflationary gravitational waves in braneworld cosmology
}

\author{
Tsutomu Kobayashi* \\ Department of Physics, Kyoto University, Kyoto 606-8502, Japan
}

(Received 17 February 2006; published 21 June 2006)

\begin{abstract}
We study the spectrum of gravitational waves generated from inflation in the Randall-Sundrum braneworld. Since the inflationary gravitational waves are of quantum-mechanical origin, the initial configuration of perturbations in the bulk includes Kaluza-Klein quantum fluctuations as well as fluctuations in the zero mode. We show, however, that the initial fluctuations in Kaluza-Klein modes have no significant effect on the late time spectrum, irrespective of the energy scale of inflation and the equation of state parameter in the post-inflationary stage. This is done numerically, using the Wronskian formulation.
\end{abstract}

DOI: $10.1103 /$ PhysRevD.73.124031

PACS numbers: $04.50 .+\mathrm{h}, 11.10 . \mathrm{Kk}, 98.80 . \mathrm{Cq}$

\section{INTRODUCTION}

The inflationary scenario predicts the existence of a gravitational wave background produced quantum mechanically [1], in much the same way as the mechanism of generating density perturbations. Detecting the inflationary gravitational wave background is a grand challenge for LISA [2], BBO [3], and other missions [4]. It will open up a new window into the very early Universe, and thus in principle the possibility of recently proposed braneworld models [5] can be tested using the inflationary gravitational waves.

A number of studies have been done aiming at obtaining a clear picture of the generation and evolution of gravitational waves in the Randall-Sundrum braneworld [6-22]. De Sitter inflation on the brane [6] is a special case where the perturbation equation is separable and exactly solvable. It predicts a flat primordial spectrum, as in fourdimensional general relativity, but the amplitude is enhanced at high energies. For more general inflation models, the tilted primordial spectrum can be calculated from the corresponding four-dimensional one by a mapping formula $[7,8]$. The late time evolution of gravitational wave perturbations at low energies is very close to that in conventional cosmology [9-11], recovering four-dimensional general relativity when the relevant length scales are larger than the bulk curvature scale $\ell$.

What is much more interesting is the evolution of gravitational waves during the high energy regime, $\ell H \gg 1$, where $H$ is the Hubble parameter. In addition to the rather trivial effect of the unconventional background expansion rate, the nontrivial effect of mode mixing may not be negligible, converting a zero mode into a Kaluza-Klein (KK) mode and vice versa efficiently at high energies. To clarify the evolution of subhorizon modes during the high energy regime, several numerical studies have been done with different numerical schemes [12-16]. Most of them concentrated on the radiation-dominated phase after inflation, and very recently Hiramatsu [16] investigated con-

*Electronic address: tsutomu@tap.scphys.kyoto-u.ac.jp sequences of a different equation of state parameter $w$. All of the above papers consider very naive initial conditions, ignoring initial abundance of KK fluctuations. However, since the gravitational wave perturbations are of quantummechanical origin, the most plausible initial configuration of the perturbations during inflation should include vacuum fluctuations in KK modes. Taking this point into account, in Refs. [8,17] Kobayashi and Tanaka have developed a numerical formulation using the Wronskian, by which one can discuss the quantum-mechanical generation and subsequent evolution of gravitational waves. Following the previous works $[8,17]$, in the present paper we revisit the spectrum of inflationary gravitational wave background in the braneworld, focusing in particular on the effect of initial abundance of the KK fluctuations.

The paper is organized as follows. In the next section we describe the background cosmological model, and then in Sec. III we review the Wronskian formulation to compute the spectrum of inflationary gravitational waves numerically. We present our numerical results in Sec. IV. Our conclusions are drawn in Sec. V.

\section{THE BACKGROUND MODEL}

We will work in the Randall-Sundrum-type braneworld [23], and the bulk metric is given by the five-dimensional anti-de Sitter spacetime

$$
d s^{2}=\frac{\ell^{2}}{z^{2}}\left(-d t^{2}+\delta_{i j} d x^{i} d x^{j}+d z^{2}\right),
$$

where $\ell$ is the bulk curvature scale. A cosmological solution can be described by a moving brane in the above static coordinates. The scale factor $a(t)$ can be expressed in terms of the location of the brane $z(t)$ as $a(t)=\ell / z(t)$, and it is governed by the modified Friedmann equation [24]

$$
H^{2}=\frac{\rho}{3 M_{\mathrm{Pl}}^{2}}\left(1+\frac{\rho}{2 \sigma}\right)
$$

where $\rho$ is the matter energy density on the brane and $\sigma=$ $6 M_{\mathrm{Pl}}^{2} / \ell^{2}$ is the tension of the brane. The matter content is characterized by the equation of state parameter 


$$
w:=\frac{p}{\rho} .
$$

Since the standard conservation law $d \rho / d \tau=-3 H(\rho+$ $p$ ) holds on the brane, we have $\rho \propto a^{-3(1+w)}$ when $w$ is constant. Using this and Eq. (2) we obtain the scale factor as a function of the proper time $\tau$ on the brane.

The initial stage of the background model is assumed to be described by a de Sitter brane with a constant expansion rate $H_{i}$, and the final stage by a Minkowski brane. The two stages are connected smoothly by a Friedmann-RobertsonWalker (FRW) brane with a constant equation of state parameter $w$. We dub this phase as the "FRW phase." The connection to the Minkowski phase is done at sufficiently low energies because we would like to focus on the possible high energy effects at the early stage of the FRW phase just after inflation. The construction here allows us to discuss the quantum-mechanical generation of gravitational waves during inflation and their subsequent evolution during the FRW phase, and to see the final amplitude of the well-defined zero mode. In the previous paper [17] only the radiation-dominated stage $(w=1 / 3)$ was considered, but in the present paper we are interested in other various values of $w$ as well.

\section{FORMULATION}

\section{A. Double null coordinates}

Let us consider gravitational wave (tensor-type) perturbations. The perturbed metric can be written as

$$
d s^{2}=\frac{\ell^{2}}{z^{2}}\left[-d t^{2}+\left(\delta_{i j}+h_{i j}\right) d x^{i} d x^{j}+d z^{2}\right],
$$

where $h_{i j}=h_{i j}(t, \mathbf{x}, z)$ is the transverse-traceless metric perturbation. We decompose it into the spatial Fourier modes as

$$
h_{i j}=\frac{\sqrt{2}}{\left(2 \pi M_{5}\right)^{3 / 2}} \int d^{3} k \phi_{\mathbf{k}}(t, z) e^{i \mathbf{k} \cdot \mathbf{x}} e_{i j},
$$

where $M_{5}$ is the fundamental mass scale which is related to the four-dimensional Planck mass $M_{\mathrm{Pl}}$ by $\ell\left(M_{5}\right)^{3}=M_{\mathrm{Pl}}^{2}$. The linearized Einstein equations give the Klein-Gordontype equation for $\phi_{\mathbf{k}}$ :

$$
\left(\frac{\partial^{2}}{\partial t^{2}}+k^{2}-\frac{\partial^{2}}{\partial z^{2}}+\frac{3}{z} \frac{\partial}{\partial z}\right) \phi_{\mathbf{k}}=0,
$$

and the junction conditions at the brane read

$$
\left.n^{a} \partial_{a} \phi_{\mathbf{k}}\right|_{\text {brane }}=0,
$$

where $n^{a}$ is the unit normal to the brane. From now on we suppress the subscript $\mathbf{k}$.

Following the previous papers $[8,17]$ we use the Wronskian formulation to compute the spectrum of gravitational waves. Basic details of the formulation and numerical scheme are explained in Ref. [8], but here we make a slight improvement. Since double null coordinates are convenient for numerical studies, our starting point is

$$
u=t-z, \quad v=t+z
$$

In this coordinate system, the trajectory of the brane can be specified arbitrarily by

$$
v=q(u)
$$

To simplify this boundary trajectory, we make a coordinate transformation

$$
U=u, \quad q(V)=v,
$$

and then the position of the brane is given by

$$
U=V
$$

The following further coordinate transformation turns out to be useful for actual numerical calculations:

$$
\begin{aligned}
& X=-\frac{\ell}{2} \ln \left(-\frac{U}{\ell}\right) \\
& T=-\frac{\ell}{2} \ln \left(-\frac{V}{\ell}\right) .
\end{aligned}
$$

Note that by an appropriate choice of the origin of the time coordinate $t$, we can always have $U<0$ and $V<0$. The position of the brane is simply given by

$$
T=X \text {. }
$$

We will perform numerical computations with equal grid spacing $\Delta$ in both $T$ and $X$ directions. The advantage of using these new coordinates is that it is easier to extend the computational domain far away from the brane in these coordinates than in the $(U, V)$ coordinates that were used in the previous studies. The step size $\varepsilon$ in the $U(V)$ coordinate increases with increasing $|X|(|T|)$, but we will adjust $\Delta$ so that the maximum of $\varepsilon$ is not so different from the step size used in Ref. [17]. Thanks to this choice of $\Delta$, the $(T, X)$ coordinates will not cause any problems in the resolution of the numerical analysis.

Now the bulk metric is written as

$$
d s^{2}=A^{2}(T, X)\left[-2 e^{-2 X / \ell} \tilde{q}^{\prime}(T) d T d X+\delta_{i j} d x^{i} d x^{j}\right],
$$

where

$$
\begin{gathered}
A(T, X):=\frac{2 \ell}{\tilde{q}(T)+\ell e^{-2 X / \ell}}, \\
\tilde{q}(T):=q\left(-\ell e^{-2 T / \ell}\right),
\end{gathered}
$$

and a prime stands for a derivative with respect to the 
argument. The proper time $\tau$ and the scale factor $a$ on the brane are given, respectively, by

$$
\begin{gathered}
d \tau=a \cdot \sqrt{2 \tilde{q}^{\prime}(T)} e^{-T / \ell} d T, \\
a=A(T, T),
\end{gathered}
$$

and hence we have

$$
\tilde{q}^{\prime}(T)=2 e^{-2 T / \ell}\left(\sqrt{1+\ell^{2} H^{2}}-\ell H\right)^{2} .
$$

Given the Hubble parameter $H$ as a function of $\tau$, one can integrate Eqs. (18) and (20) to obtain $\tilde{q}$ as a function of $T$.

In the $(T, X)$ coordinates the Klein-Gordon-type equation for $\phi$ reduces to

$$
\begin{array}{r}
2 \partial_{T} \partial_{X} \phi+\frac{3}{\tilde{q}(T)+\ell e^{-2 X / \ell}}\left[2 e^{-2 X / \ell} \partial_{T}-\tilde{q}^{\prime}(T) \partial_{X}\right] \phi \\
+e^{-2 X / \ell} \tilde{q}^{\prime}(T) k^{2} \phi=0,
\end{array}
$$

subject to the boundary condition

$$
\left.\left[\partial_{T}-\partial_{X}\right] \phi\right|_{T=X}=0 .
$$

\section{B. Wronskian formulation}

Since the system we are considering has infinitely many degrees of freedom, consisting of a zero mode and a tower of $\mathrm{KK}$ modes, it is not advisable to set up the initial value problem for the evolution of each perturbation mode. Instead, we confine our attention to a single degree of freedom concerning the zero mode in the final Minkowski phase. The necessary information that determines the amplitude of the final zero mode can be picked up by using the Wronskian. Our Wronskian evaluated on a constant $T$ hypersurface is defined by

$$
\left(\phi_{1} \cdot \phi_{2}\right):=2 i \int_{-\infty}^{T} d X A^{3}(T, X)\left(\phi_{1} \partial_{X} \phi_{2}^{*}-\phi_{2}^{*} \partial_{X} \phi_{1}\right),
$$

which is independent of the choice of the hypersurface.

Treating the graviton field $\phi$ as an operator, in the initial de Sitter phase we expand it using the annihilation and creation operators, $\hat{a}_{n}$ and $\hat{a}_{n}^{\dagger}$, as

$$
\hat{\phi}=\hat{a}_{0} \phi_{0}+\hat{a}_{0}^{\dagger} \phi_{0}^{*}+\int_{0}^{\infty} d \nu\left(\hat{a}_{\nu} \phi_{\nu}+\hat{a}_{\nu}^{\dagger} \phi_{\nu}^{*}\right),
$$

where $\phi_{n}$ and its complex conjugate $\phi_{n}^{*}$ are the mode functions in the de Sitter phase $(n=0$ for the zero mode and $n=\nu$ for the KK modes). These mode functions form a complete orthonormal basis with respect to the Wronskian:

$$
\begin{aligned}
\left(\phi_{0} \cdot \phi_{0}\right) & =-\left(\phi_{0}^{*} \cdot \phi_{0}^{*}\right)=1, \\
\left(\phi_{\nu} \cdot \phi_{\nu^{\prime}}\right) & =-\left(\phi_{\nu}^{*} \cdot \phi_{\nu^{\prime}}^{*}\right)=\delta\left(\nu-\nu^{\prime}\right), \\
\left(\phi_{0} \cdot \phi_{\nu}\right) & =\left(\phi_{0}^{*} \cdot \phi_{\nu}^{*}\right)=0,\left(\phi_{n} \cdot \phi_{n^{\prime}}^{*}\right)=0, \\
\text { for } n, n^{\prime} & =0, \nu .
\end{aligned}
$$

The index $\nu$ is related to the KK mass $m$ as

$$
m^{2}=\left(\nu^{2}+\frac{9}{4}\right) H_{i}^{2}
$$

and $\nu \geq 0$.

Similarly, in the final Minkowski phase we expand it as

$$
\hat{\phi}=\hat{A}_{0} \varphi_{0}+\hat{A}_{0}^{\dagger} \varphi_{0}^{*}+\int_{0}^{\infty} d m\left(\hat{A}_{m} \varphi_{m}+\hat{A}_{m}^{\dagger} \varphi_{m}^{*}\right),
$$

where $\varphi_{n}$ and $\varphi_{n}^{*}(n=0, m)$ are the mode functions in the Minkowski phase, and $\hat{A}_{n}$ and $\hat{A}_{n}^{\dagger}$ are the annihilation and creation operators, respectively, of their corresponding modes. These mode functions also form a complete orthonormal basis with respect to the Wronskian:

$$
\begin{aligned}
\left(\varphi_{0} \cdot \varphi_{0}\right) & =-\left(\varphi_{0}^{*} \cdot \varphi_{0}^{*}\right)=1, \\
\left(\varphi_{m} \cdot \varphi_{m^{\prime}}\right) & =-\left(\varphi_{m}^{*} \cdot \varphi_{m^{\prime}}^{*}\right)=\delta\left(m-m^{\prime}\right), \\
\left(\varphi_{0} \cdot \varphi_{m}\right) & =\left(\varphi_{0}^{*} \cdot \varphi_{m}^{*}\right)=0, \\
\left(\varphi_{n} \cdot \varphi_{n^{\prime}}^{*}\right) & =0, \\
\text { for } n, n^{\prime} & =0, m .
\end{aligned}
$$

The explicit form of the above mode functions is presented in the appendix.

Using the Wronskian, we now explain how to compute the spectrum of gravitational waves. We assume that initially the gravitons are in the de Sitter invariant vacuum state annihilated by $\hat{a}_{0}$ and $\hat{a}_{\nu}$,

$$
\hat{a}_{0}|0\rangle=\hat{a}_{\nu}|0\rangle=0 \text {. }
$$

The expectation value of the squared amplitude of the zero mode in the final Minkowski stage is

$$
\left\langle 0\left|\left(\hat{A}_{0} \varphi_{0}+\hat{A}_{0}^{\dagger} \varphi_{0}^{*}\right)^{2}\right| 0\right\rangle \simeq \frac{1}{k \ell} N_{f},
$$

where $N_{f}:=\left\langle 0\left|\hat{A}_{0}^{\dagger} \hat{A}_{0}\right| 0\right\rangle$ is the number of created zero mode gravitons and we assumed that $N_{f} \gg 1$. In deriving Eq. (30) we used the commutation relation $\left[\hat{A}_{0}, \hat{A}_{0}^{\dagger}\right]=1$ and the expression of the zero mode function (A1). The final power spectrum is then given by

$$
\mathcal{P}(k):=\frac{4 \pi k^{3}}{(2 \pi)^{3}} \frac{2}{\left(M_{5}\right)^{3}} \cdot \frac{1}{k \ell} N_{f}=\frac{k^{2}}{\pi^{2} M_{\mathrm{Pl}}^{2}} N_{f} .
$$

Note here that by multiplying the factor $2 /\left(M_{5}\right)^{3}$ we obtained the squared amplitude of the metric fluctuation, rather than that of the canonically normalized field [see the definition (5)]. 
In fact, particle production is suppressed, $N_{f} \lesssim 1$, for $k \geq k_{i}:=a_{i} H_{i}$ where $a_{i}$ is the scale factor at the end of inflation. In the present paper we are interested in the modes that exit the horizon during inflation, so that the relevant wave number is smaller than $k_{i}$.

The operator $\hat{A}_{0}$ can be projected out by making use of the Wronskian relations. Noting that the Wronskian is constant in time, we have

$$
\begin{aligned}
\hat{A}_{0} & =\left(\hat{\phi} \cdot \varphi_{0}\right)_{f}=(\hat{\phi} \cdot \Phi) \\
& =\left(\phi_{0} \cdot \Phi\right)_{i} \hat{a}_{0}+\int d \nu\left(\phi_{\nu} \cdot \Phi\right)_{i} \hat{a}_{\nu}+\text { H.c. },
\end{aligned}
$$

where $\Phi$ is a solution of the Klein-Gordon equation (21) whose final configuration is the zero mode function $\varphi_{0}$ in the Minkowski phase, and subscript $f$ and $i$ denote the quantities on the final and initial hypersurfaces, respectively. It is clear that final zero mode gravitons are created from the vacuum fluctuations both in the initial zero mode and in the KK modes:

$$
N_{f}=\left|\left(\phi_{0}^{*} \cdot \Phi\right)_{i}\right|^{2}+\int d \nu\left|\left(\phi_{\nu}^{*} \cdot \Phi\right)_{i}\right|^{2} .
$$

Correspondingly, the power spectrum (31) can be written as a sum of the two contributions:

$$
\mathcal{P}=\mathcal{P}_{0}+\mathcal{P}_{\mathrm{KK}}
$$

where

$$
\begin{gathered}
\mathcal{P}_{0}:=\frac{k^{2}}{\pi^{2} M_{\mathrm{Pl}}^{2}}\left|\left(\phi_{0}^{*} \cdot \Phi\right)_{i}\right|^{2}, \\
\mathcal{P}_{\mathrm{KK}}:=\frac{k^{2}}{\pi^{2} M_{\mathrm{Pl}}^{2}} \int d \nu\left|\left(\phi_{\nu}^{*} \cdot \Phi\right)_{i}\right|^{2} .
\end{gathered}
$$

Thus what we need to do is to solve the backward evolution of the field $\Phi$ and to evaluate the Wronskian on the initial hypersurface in the de Sitter phase, which can be done numerically $[8,17]$.

\section{INFLATIONARY GRAVITATIONAL WAVES WITH INITIAL KK FLUCTUATIONS}

Let $\delta_{T}$ be the primordial amplitude of gravitational waves from de Sitter inflation, which is given by [6]

$$
\delta_{T}^{2}=\frac{2 C^{2}\left(\ell H_{i}\right)}{M_{\mathrm{Pl}}^{2}}\left(\frac{H_{i}}{2 \pi}\right)^{2},
$$

where $H_{i}$ is the Hubble parameter during inflation and the function $C\left(\ell H_{i}\right)$ is related to the normalization of the zero mode (see the appendix). After the end of inflation the brane universe is dominated by a perfect fluid whose equation of state parameter is $w$, and the FRW phase is connected to the Minkowski phase when the energy scale of the universe becomes sufficiently low: $H=H_{0} \ll \ell^{-1}$. For the modes that reenter the horizon during the low energy regime, namely, for the long wavelength modes with $k \ll k_{*}$ where

$$
k_{*}:=a_{*} H_{*}=a_{*} / \ell,
$$

corrections to their evolution are very small, suppressed by $\ell^{2}$ and $\ell^{2} \ln \ell$ [9,11]; the mode mixing effect is inefficient and gravity on the brane is basically described by fourdimensional general relativity. Hence, for such modes the spectrum of gravitational waves is expected to have the same spectrum as the four-dimensional one:

$$
\mathcal{P}=\frac{\delta_{T}^{2}}{2}\left(\frac{k}{k_{0}}\right)^{n_{4 \mathrm{D}}}, \quad\left(k_{0}<k \ll k_{*}\right),
$$

with

$$
n_{4 \mathrm{D}}=-\frac{4}{1+3 w},
$$

where $k_{0}$ is the wave number associated with the horizon scale at the end of the FRW phase: $k_{0}:=a_{0} H_{0}$. For the relatively short wavelength modes with $k \geq k_{*}$, three nontrivial things should be considered: (i) the modified background expansion rate [Eq. (2)], (ii) the excitation of KK modes relevant at high energies, and (iii) the initial quantum fluctuations in KK modes $\mathcal{P}_{\mathrm{KK}}$. If the gravitational waves propagating in the bulk are neglected, namely, if the effects (ii) and (iii) are neglected, one would obtain an enhanced spectrum for $k \gtrsim k_{*}$,

$$
\mathcal{P}_{\uparrow} \simeq \frac{\delta_{T}^{2}}{2}\left(\frac{k_{*}}{k_{0}}\right)^{-2}\left(\frac{k}{k_{*}}\right)^{n_{\uparrow}},
$$

with

$$
n_{\uparrow}=-\frac{2}{2+3 w} .
$$

However, this evaluation will not be correct because of efficient mode mixing at high energies. ${ }^{1}$ The true spectrum will be somewhat different from the four-dimensional one and differed also from Eqs. (41) and (42). We would like to investigate how the balance of the above three things affects the spectrum.

In Refs. [13,15,16] the effects of (i) the modified Friedmann equation and (ii) the KK mode excitation have been addressed using the numerical formulations very different from ours. ${ }^{2}$ The authors of Refs. $[13,15,16]$ focused on the classical evolution of perturbations, while the initial conditions they adopt are naive, neglecting the initial abundance of KK fluctuations. In our Wronskian

\footnotetext{
${ }^{1}$ There are also braneworld models where one arrives at an interesting conclusion about cosmological gravitational waves without mode mixing [25,26].

${ }^{2}$ The formulation by Hiramatsu et al. [13] is based on the Poincaré coordinates, while Ichiki and Nakamura [15] used a single null coordinate. The two results do not agree with each other for some unclear reason. However, the Wronskian approach [17] gives the result consistent with Ref. [13].
} 
INITIAL KALUZA-KLEIN FLUCTUATIONS AND ...

formulation, the initial conditions are set quantum mechanically and hence are the most plausible. It has been (incompletely) shown that the effect of the initial quantum fluctuations in the KK modes is subdominant relative to the other two effects in the radiation-dominated phase $(w=$ $1 / 3$ ) [17]. In that case, however, the dominant two effects cancel each other and consequently we have the same spectrum as in the conventional four-dimensional universe.

In the previous paper [17] the authors failed in the precise evaluation of the fraction coming from the initial KK modes,

$$
r_{\mathrm{KK}}(k):=\frac{\mathcal{P}_{\mathrm{KK}}}{\mathcal{P}_{0}+\mathcal{P}_{\mathrm{KK}}},
$$

due to the limited number of grids in the extra direction. Now using the new coordinates $T$ and $X$, we are able to obtain much more precise values of $r_{\mathrm{KK}}$. Our refined result is shown in Fig. 1. This is different from the previous estimate by a factor of 2 or so.

To make it clear how the spectrum behaves in a more general situation, let us move on to the models with the equation of state parameter other than $1 / 3$. We have performed numerical calculations for three different cases: $w=0,2 / 3$, and 1 . The results are shown in Figs. 2-4. We find that in all the cases the modification to the expansion rate and the $K K$ mode excitation are the dominant effects, and the initial abundance of KK fluctuations gives a subdominant contribution. In contrast to the radiationdominated model, the two dominant effects do not cancel each other, yielding the spectrum different from the fourdimensional one for short wavelength modes. Assuming that the spectrum has the form

$$
\mathcal{P} \propto k^{n}, \quad k>\alpha k_{*},
$$

where we take $\alpha \sim 3$, we obtain

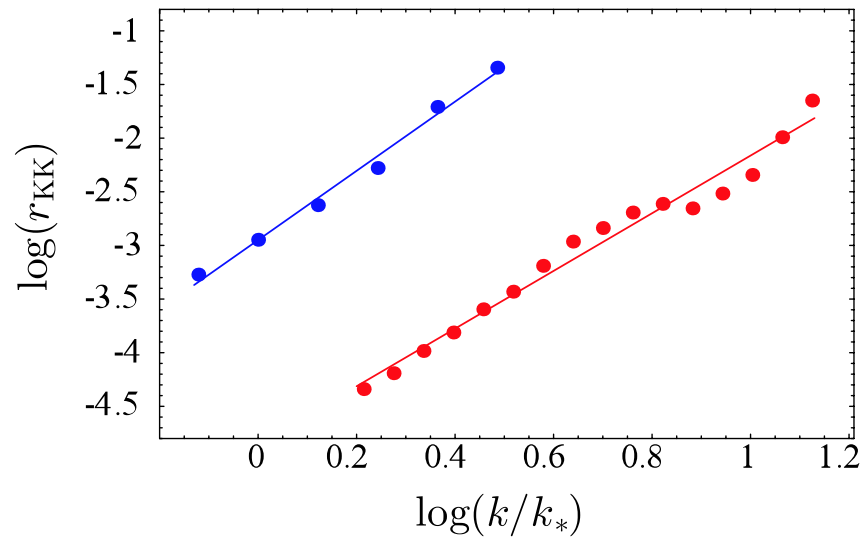

FIG. 1 (color online). Contribution of initial Kaluza-Klein fluctuations. The equation of state parameter is $w=1 / 3$ and the inflationary energy scale is given by $\ell H_{i}=100$ (lower line). $r_{\mathrm{KK}}$ is plotted also for $\ell H_{i}=10$ (upper line). (The previous estimate in Ref. [17] should be compared with the lower line.)
PHYSICAL REVIEW D 73, 124031 (2006)

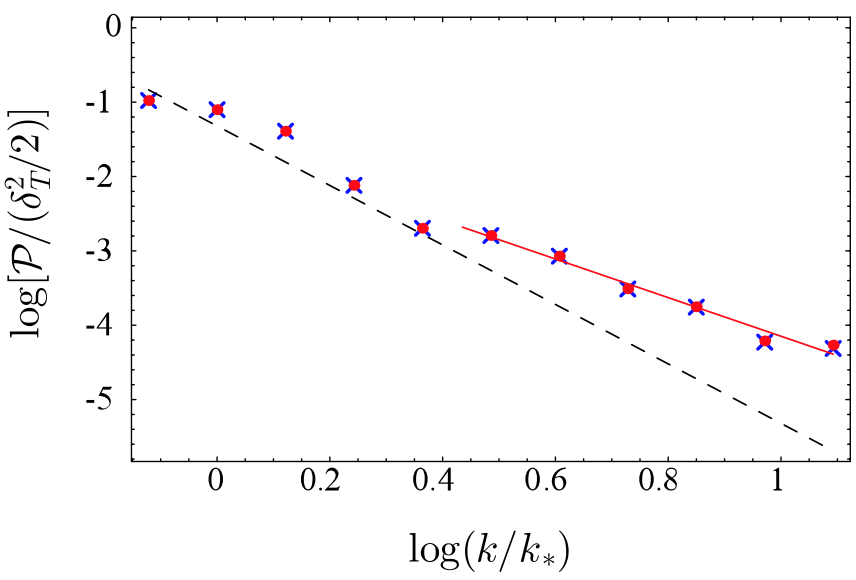

FIG. 2 (color online). Spectrum of gravitational waves from inflation with $\ell H_{i}=100$, followed by a FRW phase with $w=0$. The total power spectrum is shown by circles, while crosses represent the contribution only from the initial zero mode. Dashed line indicates the four-dimensional result.

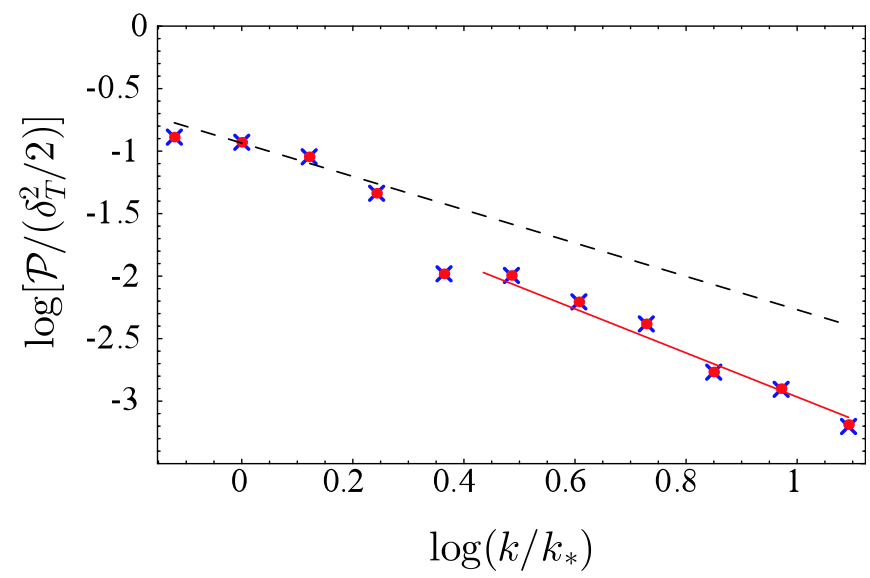

FIG. 3 (color online). Same as Fig. 2, but $w=2 / 3$.

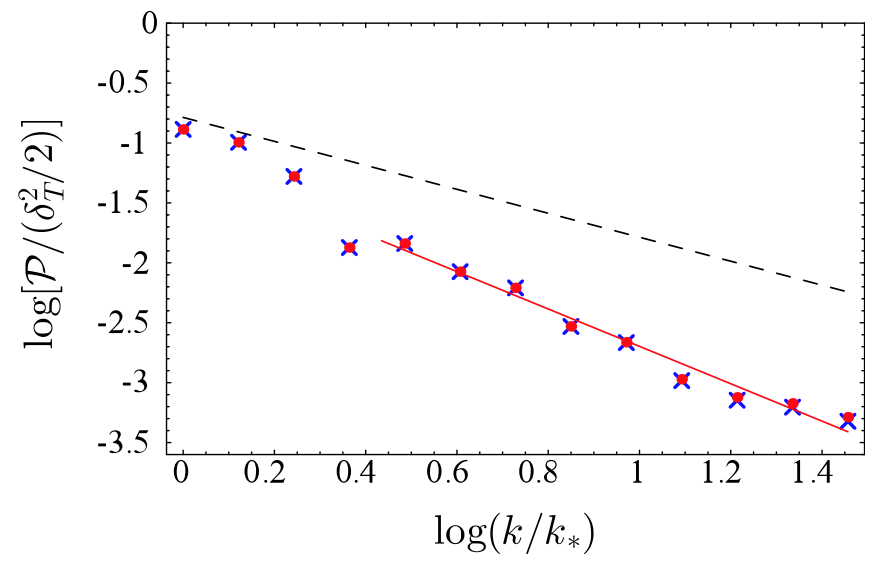

FIG. 4 (color online). Same as Fig. 2 , but $w=1$. 
TSUTOMU KOBAYASHI

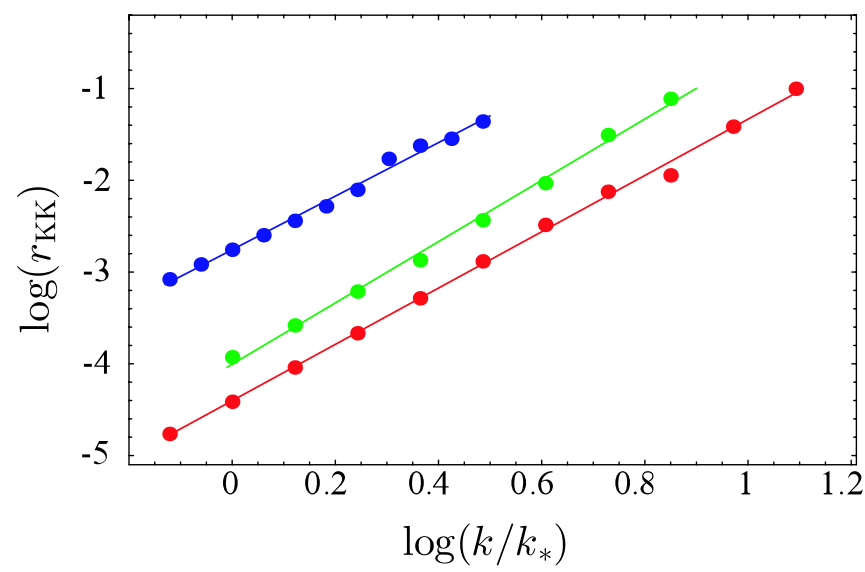

FIG. 5 (color online). Fraction $r_{\mathrm{KK}}$ for different inflationary energy scales, showing (from top to bottom) $\ell H_{i}=10,50$, and 100. The equation of state parameter is given by $w=0$.

$$
n \simeq \begin{cases}-2.6, & (w=0) \\ -1.8, & (w=2 / 3) \\ -1.6, & (w=1) .\end{cases}
$$

From this result and the previous one $(n \simeq-2$ for $w=$ $1 / 3$ ), we may deduce that the spectral tilt for short wavelength modes is given generally by

$$
n \simeq-\frac{5+3 w}{2+3 w} .
$$

This agrees with the recent numerical result by Hiramatsu [16] because of the insensitivity of the final result to the initial KK mode contamination. For the modes with $k \lesssim$ $k_{*}$, we can confirm that the spectrum is approximately given by the four-dimensional one in all the three cases.

Now we take a closer look at the contribution from the initial $\mathrm{KK}$ fluctuations. The fraction $r_{\mathrm{KK}}$ is plotted for various values of the inflationary Hubble parameter $H_{i}$ in Figs. 5 and 6 (and also in Fig. 1). While the equation of state parameter is different ( $w=0$ for Fig. 5 and $w=1$ for Fig. 6), the plots share the same feature. For fixed $k, r_{\mathrm{KK}}$

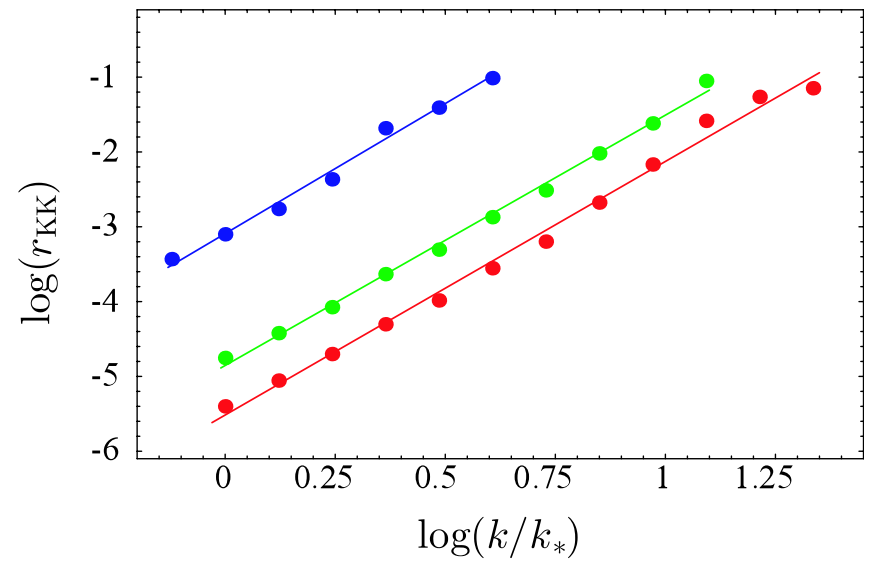

FIG. 6 (color online). Same as Fig. 5 , but $w=1$. increases with decreasing $\ell H_{i}$, but, interestingly, the maximum of $r_{\mathrm{KK}}$ is given by $\sim 0.1$ irrespective of the inflationary energy scale $\ell H_{i}$. We find that this behavior can be summarized in the following universal relation:

$$
r_{\mathrm{KK}}(k) \simeq r_{\max }\left(\frac{k}{k_{i}}\right)^{\beta}
$$

with

$$
\begin{gathered}
r_{\text {max }}<\mathcal{O}(1), \\
\beta \approx 3,
\end{gathered}
$$

and note that $k_{i}$ is given by

$$
k_{i}=a_{i} H_{i} \simeq\left(\ell H_{i}\right)^{(2+3 w) /(3+3 w)} \times k_{*} .
$$

Our numerical analysis indicates that the formula (47) holds as long as the inflationary stage lies in the high energy regime. For low energy inflation $\left(\ell H_{i} \ll 1\right) r_{\mathrm{KK}}$ is further suppressed, and we confirmed that by computing $r_{\mathrm{KK}}$ for a model with $\ell H_{i}=0.2$ and $w=1 / 3$, showing that $r_{\mathrm{KK}} \sim 10^{-3}$ at $k \sim a_{i} H_{i}$.

\section{CONCLUSIONS}

In this paper, we have investigated the spectrum of gravitational waves generated from inflation on the brane. Our analysis generalized the previous work [17] in that the present background cosmological model allows for the post-inflationary stage characterized by the equation of state parameter $w$ other than $1 / 3$. We have paid particular attention to the effect of the initial condition: the configuration of the perturbations during inflation includes vacuum fluctuations in Kaluza-Klein modes. This was made possible by the use of the Wronskian formulation [8,17], which is a distinctive point compared to the other related works.

Our numerical analysis for the various inflationary energy scale and equation of state parameter showed that the contribution of the initial KK fluctuations $r_{\mathrm{KK}}(k)$, defined in Eq. (43), always behaves as Eq. (47), implying that the initial KK fluctuations give rise to the subdominant effect on the spectrum. This is the main conclusion of the paper, being true in the more general setup than the previous work [17]. The present calculation covers the limited range of the inflationary energy scale (up to $\ell H_{i}=100$ ), but we believe the results to be sufficient for concluding that also for higher energy scales, $r_{\mathrm{KK}}$ is smaller than order of unity even at the smallest wavelength concerned $\left(k \sim k_{i}\right)$. While the effect of the initial KK fluctuations can be safely neglected, the enhancement effect due to the modified Friedmann equation and the damping effect due to the excitation of KK modes are dominantly work, yielding the spectral tilt $n \simeq-(5+3 w) /(2+3 w)$ for short wavelength modes. This can be an observational signature of the braneworld model with nonstandard (i.e., $w \neq 1 / 3$ ) cosmological history. 
All the results in this paper are given numerically, but surely they will be great help for understanding the evolution of gravitational wave perturbations in the braneworld. Now the clues are in order: the spectral index is given by Eq. (46) and it is insensitive to the contamination of the initial KK fluctuations, with their effect expressed in detail by Eqs. (47)-(49). We will hopefully provide some analytic arguments complementary to the present numerical results in a future publication.

\section{ACKNOWLEDGMENTS}

I would like to thank Takahiro Tanaka for useful comments. I am supported by the JSPS under Contract No. 01642.

\section{APPENDIX: MODE FUNCTIONS}

In this appendix, we present an explicit form of the mode functions in the Minkowski and de Sitter braneworlds.

In the Minkowski braneworld, the normalized zero mode function is given by

$$
\varphi_{0}(t)=\frac{1}{\sqrt{2 k \ell}} e^{-i k t}
$$

while the normalized KK mode function is

$$
\varphi_{m}(t, z)=\frac{1}{\sqrt{2 \omega \ell^{3}}} e^{-i \omega t} u_{m}(z),
$$

with

$$
u_{m}(z):=z^{2} \sqrt{\frac{m}{2}} \frac{Y_{1}(m \ell) J_{2}(m z)-J_{1}(m \ell) Y_{2}(m z)}{\sqrt{\left[Y_{1}(m \ell)\right]^{2}+\left[J_{1}(m \ell)\right]^{2}}},
$$

and

$$
\omega=\sqrt{k^{2}+m^{2}}
$$

In the de Sitter braneworld we introduce new coordinates $(\eta, \xi)$, which are related to $(t, z)$ as

$$
t=\eta \cosh \xi+t_{0}, \quad z=-\eta \sinh \xi,
$$

where $t_{0}$ is an arbitrary constant. In $(\eta, \xi)$ frame the de Sitter brane is located at a fixed coordinate position $\xi=$ $\xi_{b}=$ constant, and the Hubble parameter on the brane is given by $H_{i}=\ell^{-1} \sinh \xi_{b}$. The normalized zero mode is

$$
\phi_{0}(\eta)=C\left(\ell H_{i}\right) \cdot \frac{H_{i}}{\sqrt{2 k \ell}}\left(\eta-\frac{i}{k}\right) e^{-i k \eta},
$$

with

$$
C(x):=\left[\sqrt{1+x^{2}}+x^{2} \ln \left(\frac{x}{1+\sqrt{1+x^{2}}}\right)\right]^{-1 / 2},
$$

and the $\mathrm{KK}$ mode functions are found in the form of $\phi_{\nu}(\eta, \xi)=\psi_{\nu}(\eta) \chi_{\nu}(\xi)$, where

$$
\begin{gathered}
\psi_{\nu}(\eta)=\frac{\sqrt{\pi}}{2} \ell^{-3 / 2} e^{-\pi \nu / 2}(-\eta)^{3 / 2} H_{i \nu}^{(1)}(-k \eta), \\
\chi_{\nu}(\xi)=C_{1}(\sinh \xi)^{2}\left[P_{-1 / 2+i \nu}^{-2}(\cosh \xi)\right. \\
\left.-C_{2} Q_{-1 / 2+i \nu}^{-2}(\cosh \xi)\right]
\end{gathered}
$$

with

$$
\begin{gathered}
C_{1}=\left[\left|\frac{\Gamma(i \nu)}{\Gamma(5 / 2+i \nu)}\right|^{2}+\mid \frac{\Gamma(-i \nu)}{\Gamma(5 / 2-i \nu)}\right. \\
\left.-\left.\pi C_{2} \frac{\Gamma(i \nu-3 / 2)}{\Gamma(1+i \nu)}\right|^{2}\right]^{-1 / 2}, \\
C_{2}=\frac{P_{-1 / 2+i \nu}^{-1}\left(\cosh \xi_{b}\right)}{Q_{-1 / 2+i \nu}^{-1}\left(\cosh \xi_{b}\right)} .
\end{gathered}
$$

[1] For a review, see, e.g., M. Maggiore, Phys. Rep. 331, 283 (2000).

[2] http://lisa.jpl.nasa.gov/.

[3] Phinney et al., NASA Mission Concept Study (2003).

[4] See, e.g., N. Seto, S. Kawamura, and T. Nakamura, Phys. Rev. Lett. 87, 221103 (2001).

[5] For a review, see, e.g., R. Maartens, Living Rev. Relativity 7, 7 (2004)

[6] D. Langlois, R. Maartens, and D. Wands, Phys. Lett. B 489, 259 (2000).

[7] T. Kobayashi, H. Kudoh, and T. Tanaka, Phys. Rev. D 68, 044025 (2003).

[8] T. Kobayashi and T. Tanaka, Phys. Rev. D 71, 124028 (2005).
[9] T. Tanaka, gr-qc/0402068.

[10] K. Koyama, J. Cosmol. Astropart. Phys. 09 (2004) 010.

[11] T. Kobayashi and T. Tanaka, J. Cosmol. Astropart. Phys. 10 (2004) 015.

[12] T. Hiramatsu, K. Koyama, and A. Taruya, Phys. Lett. B 578, 269 (2004).

[13] T. Hiramatsu, K. Koyama, and A. Taruya, Phys. Lett. B 609, 133 (2005).

[14] K. Ichiki and K. Nakamura, Phys. Rev. D 70, 064017 (2004).

[15] K. Ichiki and K. Nakamura, astro-ph/0406606.

[16] T. Hiramatsu, Phys. Rev. D 73, 084008 (2006).

[17] T. Kobayashi and T. Tanaka, Phys. Rev. D 73, 044005 (2006). 


\section{TSUTOMU KOBAYASHI}

[18] D. S. Gorbunov, V. A. Rubakov, and S. M. Sibiryakov, J. High Energy Phys. 10 (2001) 015.

[19] R. Easther, D. Langlois, R. Maartens, and D. Wands, J. Cosmol. Astropart. Phys. 10 (2003) 014.

[20] R. A. Battye, C. Van de Bruck, and A. Mennim, Phys. Rev. D 69, 064040 (2004).

[21] R. A. Battye and A. Mennim, Phys. Rev. D 70, 124008 (2004).

[22] C. Cartier, R. Durrer, and M. Ruser, Phys. Rev. D 72, 104018 (2005).

[23] L. Randall and R. Sundrum, Phys. Rev. Lett. 83, 4690
(1999).

[24] P. Binetruy, C. Deffayet, and D. Langlois, Nucl. Phys. B565, 269 (2000); P. Binetruy, C. Deffayet, U. Ellwanger, and D. Langlois, Phys. Lett. B 477, 285 (2000); S. Mukohyama, Phys. Lett. B 473, 241 (2000); D. Ida, J. High Energy Phys. 09 (2000) 014; P. Kraus, J. High Energy Phys. 12 (1999) 011.

[25] M. Cavaglia, G. De Risi, and M. Gasperini, Phys. Lett. B 610, 9 (2005).

[26] G. De Risi, gr-qc/0601132. 